\title{
Ueber das Vorkommen des Trimethylamins in der Heringslake.
}

Werthheim erhielt zuerst die Base $\mathrm{C}^{6} \mathrm{H}^{9} \mathrm{~N}$, und beschrieb sie als Propylamin; er fand sie unter den Zersetzungsproducten des Narkotins durch Kalk, in der Heringslake, und Anderson unter den Zersetzungsproducten des Codeins. Hoffmann wies zuerst darauf hin, dass das Propylamin vielleicht Trimethylamin sei. Offenbar kann $\mathrm{C}^{6} \mathrm{H}^{9} \mathrm{~N}$ eine von den drei folgenden Basen sein.

Trimethylamin. Methyl-Aethylamin.
$\mathrm{C}^{2} \mathrm{H}^{3}$
$\mathrm{C}^{2} \mathrm{H}^{3} \mathrm{n}$
$\left.\mathrm{C}^{2} \mathrm{H}^{3}\right)$
$\mathrm{H}$
$\mathrm{C}^{2} \mathrm{H}^{3}$

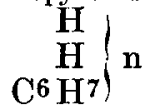

Propylamin.

Aus G. H. Winkles neuester Untersuchung geht aber hervor, dass die aus der Heringslake dargestellte Base nicht Propylamin, sondern Trimethylamin ist. (Quat. Journ. of the chem. Soc. of Lond. - Chem.-pharm. Centrbl. 1854. Nr. 32.)

$B$.

\section{Chemische Untersuchungen über die Knochen.}

Die Resultate der neuesten Untersuchung Fremy's über die Knochen sind folgende:

1) Die organische in den Knochen enthaltene Substanz, die Robin und Verdeil in neuester Zeit mit dem Namen Ossein bezeichnet haben, ist isomer mit dem Leim. Die Umwandlung des Osseins in Leim lässt sich mit der Verwandlung der Stärke und der Cellulose in Dextrin vergleichen, sie geht auch unter denselben Umständen vor sich, die Wirkung der Säuren unterstützt dieselbe.

2) Das Ossein aus den Knochen junger Thiere hat dieselbe Zusammensetzung wie das von älteren, verwandelt sich aber leichter in Leim.

3) Das Ossein verschiedener Thierclassen, Säugethiere, Vögel, Reptilien, Fische, hat dieselbe Zusammensetzung.

Ausser dem Ossein kommt aber in der Knochensubstanz noch ein organischer Körper vor, der sich durch Behandeln mit siedendem Wasser nicht in Leim verwandelt, übrigens mit dem Ossein isomer ist. Dieser wurde in den Knochen gewisser Wasservögel und den Gräten einiger Fische angetroffen, er bildet eine weisse, durch- 
sichtige, elastische Masse, die, wenn man sie mit Anwendung von Säure darstellt, die Form der Knochen behält.

4) Das Ossein ist, wie es scheint, in den Knochen frei vorhanden, es ist nicht mit dem phosphorsaurem Kalke chemisch verbunden.

5) Der phosphorsaure Kalk der Knochen ist der dreibasisch-phosphorsaure Kalk; Fremy's Untersuchungen führten also zu demselben Resultate, wie die anderer Chemiker in neuerer Zeit.

6) Macht es E. Fremy wahrscheinlich, dass die phosphorsaure Ammoniaktalkerde einen Bestandtheil der Knochen ausmacht.

7) Das Fluorcalcium kommt auch in geringer Menge allgemein in den Gebeinen vor.

8) Hinsichtlich der Constitution und der Entwickelungsweise der Knochen zeigt Fremy, dass an einem und demselben Knochen der dichte Theil von dem schwammigen verschieden ist, der erstere enthält mehr Kalksalze als der letztere.

9) Aus den Arbeiten von Flourens weiss man, dass das z̈ussere Periosteum eines Knochens die Knochensubstanz ununterbrochen secernirt, während sie im Innern resorbirt wird, es findet daher im Knochen eine ununterbrochene Störung statt. Die Analysen Fremy's weisen nun auch nach, dass die älteren Knochenschichten eine andere $\mathrm{Zu}$ sammensetzung haben, als die jüingeren.

Die folgenden Resultate der Untersuchung lehren ferner, dass das Alter keine grosse Verschiedenheiten in der Zusammensetzung der Knochen bedingt.

10) Die Knochen eines Fötus enthielten fast ebenso viel Kalksalze wie die eines Greises. Die ersten verknöcherten Puncte, die im Knorpel eines Fötus auftreten, haben dieselbe Zusammensetzung wie die im Knorpel der Erwachsenen sich bildenden. Der verknöcherte Theil, der sich an der Stelle eines Knochenbruches neu ausbildet, hat dieselbe Zusammensetzung wie der Knochen, der brach.

Hieraus geht hervor, dass der Knochen sich nicht nach und nach durch eine Aufeinanderfolge von Kalksalzen in der Knorpelsubstanz ausbildet, er bildet sich vielmehr durch das Auftreten und Aneinanderlegen von Knochenpuncten, die, wenn man sie für sich betrachtet, in ihrem ersten Erscheinen, sei es im Knorpel des Fötus oder in der bei Heilung eines Knochenbruches neu sich bildenden Knochenlöthung schon dieselbe Zusammensetzung 
haben wie in einem ganz ausgebildeten Knochen. Die Thatsache, dass die Gebeine des Greises leichter brechen, als die eines jüngeren Mannes, ist deshalb auch nicht so zu erklären, dass erstere weniger Knorpel und mehr Kalksalze enthalten, der Grund ist vielmehr der, dass im Knochen des Greises der dichte Theil des Knochens eine geringere und der schwammige Theil eine grössere Menge desselben ausmacht, und dass der Knochen des Mannes, weil er wasserreicher, auch elastischer ist als der des Greises.

11) Ueber das Verhältniss der Mengen von kohlensaurem Kalke zum phosphorsauren der Knochen und der Kalkgebilde des Thierreiches im Allgemeinen ergaben Fremy's Analysen Folgendes: In don ausgebildeten Knochen der Wirbelthiere beträgt der Gehalt an phosphorsaurem Kalke selten über 64, der an kohlensaurem 10 Proc. Das Verhältniss zwischen diesen Salzen ist ziemlich constant und entspricht fast dem von 1 Aeq. kohlensauren Kalks auf 3 Aeq. phosphorsauren. Der kohlensaure Kalk scheint mit dem Alter der Knochen etwas zuzunehmen. Die phosphorsaure Talkerde beträgt gegen 2 Proc. Dic Tabelle, die Fremy in seiner $\mathrm{Ab}$ handlung entworfen hat, zeigt, dass die Knochen des Menschen und die von Thieren sehr verschiedener Classen von Wirbelthieren oftmals ganz gleiche Zusammensetzungen haben. Eine solche Uebereinstimmung zeigt sich bei den Knochen des Menschen, Elephanten, Rhinoceros, Rindviehes, der Ziege, des Kaninchen, Löwen, Pottfisch, Strauss, der Schlange, Schildkröte, des Kabeljau, der Barbe u. a. Fremy nimmt an, dass der Knochen so verschiedener Thierfamilien immer dieselbe Bedingung zu crfüllen habe, er soll stets eine beträchtliche Härte und Festigkeit und muss somit dieselbe Zusammensetzung haben.

12) Dayegen finden sich Verschiedenheiten in den Knochen verschiedener Species einer und derselben Thierclasse. So sind die Knochen von einem herbivoren Säugethiere stets reicher an Kalksalzen als bei einem carnivoren. Die Knochen der Reptilien haben dieselbe Zusammensetzung wie die der carnivoren Säugethiere. Die Knochen der Vögel sind reicher an Kalksalzen als die der fleischfressenden Säugethiere.

13) Die Gräten von Grätenfischen, vom Karpfen, Hecht, haben dieselbe Zusammensetzung, wie die Knochen von Säugethieren; die von Knorpelfischen enthalten 
weniger Kalksalze als jene. Der Knorpel der Lamprete enthält fast gar keine Mineralbestandtheile, und kann also auch nicht ferner für einen Knochen gehalten werden.

14) Die Fischschuppen haben eine grosse Aehnlichkeit mit dem Knochen und Knorpel der Fische. Manche enthalten bis 60 Proc. an Kalksalzen; andere, wie die vom Karpfen, enthalten nur 35 Proc. Die Kalksalze sind von derselben Natur wie die in den Knochen. Die organische Materie verwandelt sich, wie die der Knochen, in Leim und hat auch dieselbe Znsammensetzung.

15) Die Analysen von fossilen Knochen haben verschiedene Resultate gegeben, in manchen ist die organische Substanz mehr oder weniger vollständig durch verschiedene Mineralbestandtheile ersetzt, die je nach dem Terrain kohlensaurer, schwefelsaurer und flusssaurer Kalk, oder Kieselsïure als Quarz sind. Auch finden sich darin Spurcn von Eisen. Manche Knochen enthielten 20 Proc. Ossein, andere nichts mehr davon. Das fossile Ossein verwandelt sich durch siedendes Wasser in Leim. Bei den fossilen Knochen findet man die eingedrungenen Mineralbestandtheile viel mehr in den schwammigen Theilen, als in den dichten.

In den Geweihen der Wiederkäuer findet man bezüglich der Kalksalze dasselbe Verhalten wie bei den Knochen. In den jungen weniger, in den älteren mehr Kalksalze.

Bei Untersuchung der Zähne fand Fremy, dass das Email nur 2-3 Proc. organische Materie, 3-4 Proc. kohlensauren Kalk, Spuren von Fluorcalcium und gegen 90 Proc. phosphorsauren Kalk enthielt, während das Innere genau dieselbe Zusammensetzung hatte wie die Knochen.

16) Die Kalkconcretionen, welche die Arterien der Greise ossificiren und schon oft mit Knochen verglichen wurden, enthalten nach Fremy's Untersuchungen dieselben Kalksalze wie die Knochen, welche auch in derselben Weise mit der Knocherısubstanz vereinigt sind. Statt des Osseins aber findet sich hier eine Substanz von eiweissartiger Natur, wodurch diese Concretionen von den Knochen wesentlich verschieden sind.

17) Von Zoophyten hat Fremy Pennatula analysirt. Ihre Kalkgebilde haben einige Aehnlichkeit mit Knochen, denn sie enthalten einen organischen und einen mineralischen Theil, der in phosphorsaurem und kohlensaurem Kalke besteht. Sie weichen aber hinsichtlich der organi- 
schen Substanz, die in Säuren unlöslich ist, und der Proportion der Kalksalze von cinander ab. Der kohlensaure Kalk ist darin in grösserer Menge als in den Knochen, und der phosphorsaure, der in den Knochen doch auf 60 Proc. steigt, erreicht höchstens die Menge von 24 Proc.

18) Hinsichtlich der Muschelschalen kam Fremy zu keinem neuen Resultate, diese Gebilde können nicht mit den Knochen verglichen werden; sie bestehen meist aus kohlensaurem Kalke und enthalten nur Spuren von phosphorsaurem; die Substanz, welche den Muschelschalen die Färbung ertheilt, ist stickstofthaltig, Säure und höhere Temperatur zerstört sie sogleich, sie ist identisch mit der Substanz, die die Korallen roth färbt.

Die Muschelschalen erhalten aber noch eine andere Substanz in reichlicher Menge, die vom Ossein verschieden ist. Sie verwandelt sich durch siedendes Wasser nicht in Leim. Fremy will sie genauer untersuchen und giebt ihr vorläufig den Namen Conchiolin.

19) Das Skelett der Crustaceen hat in physiologischer Hinsicht keine Analogie mit der Knochensubstanz, Frem y hat aber einige auf ihre Kalksalze geprüft und beabsichtigte, die stickstofffreie Substanz, welcher man den Namen Chitin gegeben hat und die auch bei den Insekten gefunden wird, zu untersuchen.

Der unorganische Theil, der sich sehr unregelmässig in den Hülsen der Crustaceen absetzt, besteht in phosphorsaurem und kohlensaurem Kalk. Der phosphoršaure Kalk beträgt nie mehr als 6-7 Proc. Die Analysen von Chitin führen zu der Zusammensetzung der Cellulose, doch giebt es mit Salpetersäure kein Pyroxylin und ist also hierdurch von jener als verschieden zu betrachten.

20) Die stickstoffhaltigen Substanzen des Horns, des Schildplatts, des Fischbeins sind von der organischen Materie der Knochen verschieden. Sie geben beim Kochen keinen Leim. (Compt. rend.T. 39. - Chem.-pharm. Centrbl. 1855. $N r .5$. 